\title{
GAIA Gestational Diabetes Mellitus Level of Diagnostic Certainty
}

National Cancer Institute

\section{Source}

National Cancer Institute. GAIA Gestational Diabetes Mellitus Level of Diagnostic

Certainty. NCI Thesaurus. Code C128671.

A classification of maternal and fetal outcomes relating to gestational diabetes mellitus, developed by the Global Alignment of Immunization safety Assessment in pregnancy, based on the extent to which the diagnosis has been confirmed. 